\title{
Intraoperative Investigation of Vibration and Acoustic Characteristics of Venous Pulsatile Tinnitus Using Confocal Laser Displacement Sensor, Doppler Ultrasonography, and Piezoelectric Film Sensor: A Pilot Study
}

\author{
Yue-Lin Hsieh ${ }^{1,2,3}$ and Wuqing Wang 2,3* \\ ${ }^{1}$ Fudan University, 220 Handan Rd., Yangpu Dist., Shanghai 200433, China \\ ${ }^{2}$ Department of Otology and Skull Base Surgery, Eye, Ear, Nose, \& Throat Hospital, Fudan University, \\ 83 Fenyang Rd., Xuhui Dist., Shanghai 200031, China \\ ${ }^{3}$ NHC Key Laboratory of Hearing Medicine, 83 Fenyang Rd., Xuhui Dist., Shanghai 200031, China
}

(Received December 31, 2020; accepted February 26, 2021)

Keywords: pulsatile tinnitus, vibration, sensor, acoustic, water occlusion test

The vibrations of a thinned sigmoid plate and exposed sinus vessel wall in patients with sigmoid sinus wall anomalies (SSWAs) have long been speculated to be a pivotal cause of venous pulsatile tinnitus (PT). This is the first in vivo study to investigate whether vibrations resulting from SSWAs induce PT using multiple sensor systems. A confocal laser displacement sensor system was intraoperatively deployed to gauge displacements of the focal anatomical structure in 12 subjects with venous PT with or without SSWAs. Doppler ultrasonography was performed to detect the blood flow and psychoacoustic characteristics of PT. Computational fluid dynamics (CFD) and piezoelectric sensors were deployed to assess the relationship between the vessel wall pressure and hemodynamics. The ultrasonographic examination showed that the PT pitch matched the blood flow sampled at the center of the sinus lumen in all subjects and that there was no statistical difference between the displacements of the sigmoid plate and vessel wall before separation, indicating that PT is strongly associated with flow-induced noise rather than vibration-induced noise. In addition, the quantitative water occlusion test can be potentially indicative for the surgical and mechanistic study of PT.

\section{Introduction}

The sigmoid plate (sigmoid sinus wall) is an osseous structure that overlies the surface of the sigmoid sinus. While setting apart the sigmoid sinus and airy honeycomb-like mastoid cavity, this solid sigmoid plate is deemed to preclude the vascular sounds in the posterior fossa from transmitting to the inner ear. ${ }^{(1-6)}$ Unfortunately, in some individuals, the sigmoid plate is not intact. A growing number of clinical studies have indicated a strong possibility that the unremitting transverse sinus jet-flow impingement erodes the sigmoid plate..$^{(1,2,7,8)}$ Without the shield of the overlying solid osseous wall, the sinus vessel wall protrudes into the mastoid air

*Corresponding author: e-mail: wwuqing@eent.shumu.edu.cn https://doi.org/10.18494/SAM.2021.3284 
cells under the transverse sinus flow impingement, which over time forms a diverticulum. The sigmoid sinus wall dehiscence and diverticulum are now known as sigmoid sinus wall anomalies (SSWAs). This theory of mechanically induced sigmoid plate erosion was first proposed by Eisenman back in 2011,(2) and has been speculated to be one of the most important causative factors of venous pulsatile tinnitus (PT).

Venous PT is the self-perception of the vascular sound derived from the dural venous sinus..$^{(1,5-9)}$ It is estimated that approximately 20 to $48 \%$ of venous PT patients present SSWAs. ${ }^{(9,10)}$ Through extrapolation of surgical successes, PT associated with a dehiscent sigmoid plate is hypothesized to emerge under two major scenarios (see also Fig. 1). Theory A: the intrasinus flow-induced sound "permeates" through the boney defect and propagates via an air-conduction transmission route to the cochlea, and Theory $B$ : the vascular wall without the overlying sigmoid plate vibrates, and the generated vibrational sound and vibrotactile stimulation are transmitted to the cochlea via bone conduction. ${ }^{(1,2,7,10-16)}$ Despite the uncertainty in how PT is mechanically generated (the amplitude of vascular sounds surpassing a baseline hearing threshold), both resurfacing the sigmoid wall using coupled layers of soft and hard materials to

(a)

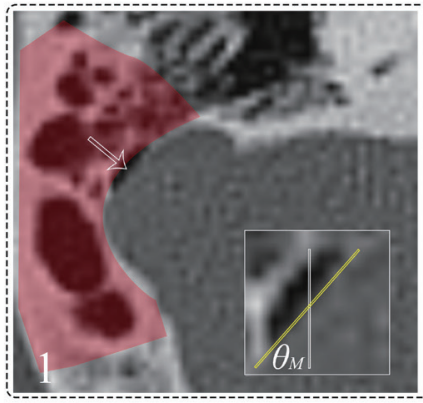

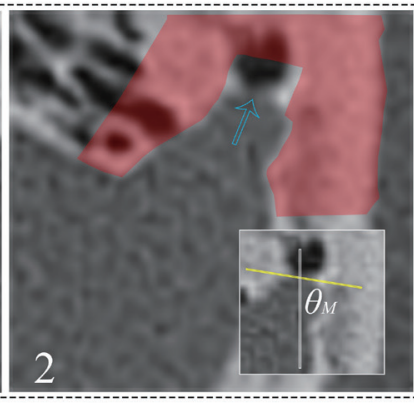

3
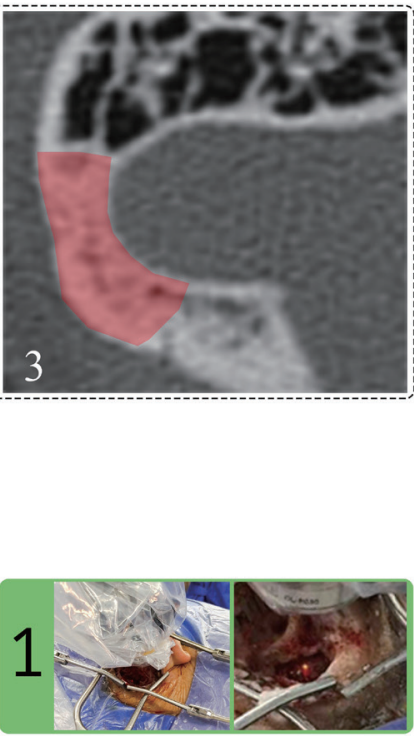

(b)

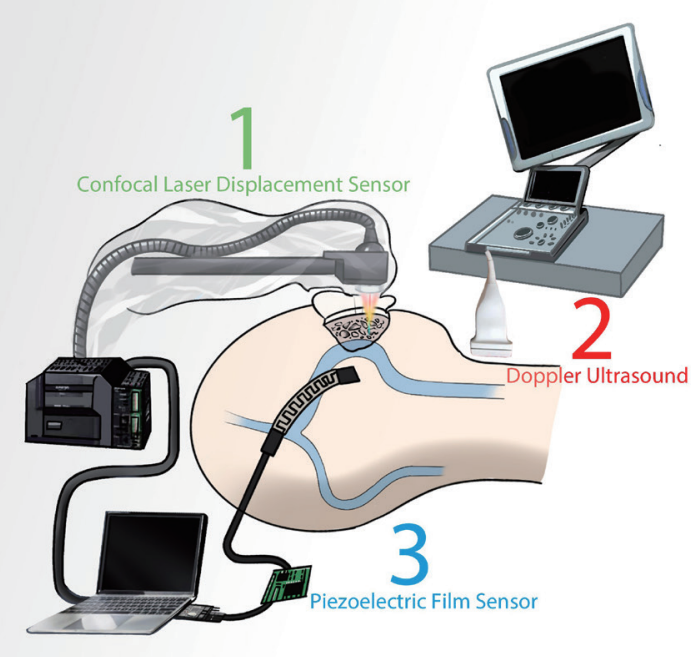

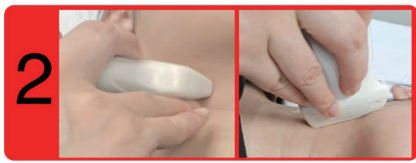

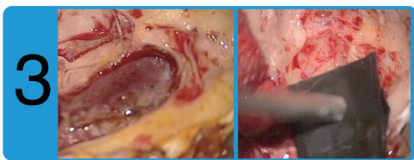

Fig. 1. (Color online) Schematic diagram showcasing the intraoperative establishment of multiple sensor systems and preoperative ultrasonographic Doppler. (a) Radiologic data of the categorized groups: dehiscence (1, white arrow), diverticulum (2, blue arrow), and non-SSWA (3) groups. The marked red area indicates the area of mastoid bone obliteration for sensor measurements. $\theta_{M}$ indicates the measuring angle under the sensor head for the dehiscence and diverticular groups. (b) Schematic graph demonstrating the setup of the sensor systems. 
baffle the venous flow sound and altering the intrasinus hemodynamics via extraluminal/ endoluminal surgical techniques have shown high surgical success rates. ${ }^{(7,17)}$

As a byproduct secondary to flow impaction, the flow-induced vibration of anatomical structures may not be the only mechanical source of PT. Venous PT can occur in patients with an intact sigmoid plate (without SSWAs). ${ }^{(7,13,18)}$ It is suspected that the fluid-borne sound generated from vortical flow structures with increased flow inertia is another potential, if not a more dominant, causative factor of $\mathrm{PT} .{ }^{(8)}$ Nevertheless, in vitro studies have strongly indicated that vibrations of anatomical structures serve as the primary cause of PT. ${ }^{(19)}$ This raises two fundamental questions: 1) If the vibration of the focally exposed vessel wall significantly exceeds that of the juxta-dehiscent structures, will it be a dominant causative factor of PT? 2) If the entire sigmoid plate vibrates, will it serve as the dominant causative factor of PT, as opposed to the vibration of the exposed vessel wall alone?

The current predicament, however, lies in the fact that the in vitro experimental and computational fluid dynamics (CFD) studies fall short of reproducing the realistic anatomical structures. For instance, less than half of the soft and collapsible vascular surface attaches to the solid sigmoid plate, with the remainder resting against soft brain tissues. ${ }^{(20)}$ Such unique anatomical settings render the realistic setup of boundary conditions excruciatingly challenging. It is further complicated by the fact that the dural venous sinus is wrapped around by the periosteal and meningeal layers of dura mater made of dense irregular connective tissue. ${ }^{(20)}$ Beyond that, the sigmoid sinus is tightly adhered to the sigmoid groove. Mild or occasionally strong pulsations can only be eyeballed when the epidural space is exposed, and, interestingly enough, PT persists upon precluding or allowing the displacement of the vascular wall. ${ }^{(7)}$ This empirical observation lends to further speculation of whether the invisible vibration of a small area of the vessel wall or sigmoid plate causes audible PT or whether there is no detectable vibration at all.

To date, there has been no documentation on the vibrational patterns of the sigmoid plate and sinus vascular wall. The goals of this study were to gauge the vibrational patterns of the sigmoid plate/sinus vessel wall and whether they contribute to PT. Through the implementation of various sensor systems, one of the long-speculated mechanical sources of PT, i.e., the vibration of the sigmoid plate/sinus vessel wall, is objectively justified. These data should provide a deeper understanding of the mechanistic aspects of PT.

\section{Materials and Methods}

\subsection{Radiologic/clinical data and characterization of anatomical geometry}

For this study, we recruited 12 persistent venous PT patients presenting transverse-sigmoid sinus enlargement with or without SSWAs from May 2019 to January 2021. They were subdivided into three groups: 1 ) dehiscence ( $n=5$, cases 1 to 5), diverticulum ( $n=4$, cases 6 to 9 ), and non-SSWA ( $n=3$, cases 10 to 12 ) groups. The definition of transverse-sigmoid sinus enlargement and SSWAs was in line with previous studies. ${ }^{(1,7)}$ Transverse-sigmoid sinus enlargement was defined as the ipsilesional transverse-sigmoid sinus $150 \%$ larger than that of 
the contralesional side. Dehiscence (subjects with a dehiscent sigmoid plate) was defined as at least three consecutive axial high-resolution computed tomography (HRCT) cuts without a boney plate overlying the sigmoid sinus and protrusion into the sinus wall. Since dehiscence may not be confirmed solely via radiological means, the final grouping of participants with dehiscence was determined by intraoperative observation. A diverticulum was defined as a protrusion of the sinus vessel wall into mastoid air cells or the mastoid cortex, forming a pouchlike irregular vessel contour. Those with a diverticulum protruding into the mastoid cortex without entering the mastoid cavity were excluded from this study. The diameter, area, and angular measurements of HRCT and magnetic resonance (MR) angiography/venography were performed using a NUMARIS/4 workstation (SYNGO MRB17, Siemens AG, Germany) and Mimics 19.0 (Materialise, Belgium). All participants responded to diagnostic jugular compression, indicating that their PT originated from extradural venous sinus. No other aberrant vascular anomalies or radiological/clinical signs related to increased intracranial hypertension were found in all participants. The other medical history of all participants was insignificant.

All participants in this study provided written informed consent, and all experimental measurements were conducted in accordance with the Declaration of Helsinki and its later amendments or comparable ethical standards. Ethics approval for this study was obtained from the Ethical Committees of the Eye, Ear, Nose, \& Throat Hospital in Shanghai, China.

\subsection{Intraoperative confocal laser displacement sensor system and measurement design}

The adopted surgical procedure was similar to the previously described extraluminal surgical technique performed. ${ }^{(7,8,18)}$ A CL-P030N ultracompact confocal multicolor laser displacement sensor (Keyence, Japan) with $0.25 \mu \mathrm{m}$ resolution was deployed during the extraluminal compression surgery shown in Fig. 1. The head of participants with SSWAs was inclined by the angle between the vertical line bisecting the connecting line of the dehiscent ends and the perpendicular line $\left(\theta_{M}\right)$. For those without SSWAs, the laser spot was focused on the vascular surface at the most prominent transverse-sigmoid junction after skeletonization without invading the mastoid air cells. The IP67 waterproof sensor head attached to a stand was angled $90^{\circ}$ downward and was disinfected twice pre- and intra-operatively using $2.5 \%$ iodine tincture and then $75 \%$ alcohol for deiodination. Except for the bottom surface of the sensor head, all parts were covered by a sterile sleeve. A wide-angle lensing sensor head allowing high-accuracy measurement on curved surfaces was placed $30 \mathrm{~mm}$ directly above the sinus vessel wall. The high-precision linear measurement range was $\pm 0.72 \mu \mathrm{m}$. A Quad-Spot system with $38 \mu \mathrm{m}$ spot diameter directed light onto four points on the target, which was received by four separate highresolution CMOSs. The sampling cycle was set at $1000 \mu$ s and all detected signals were recorded for at least 20 pulsating cycles using CL-NavigatorN 1.4.0.0 software (Keyence, Japan). The discrete-time short-time Fourier transform [Eq. (1)] and variational mode decomposition (VMD) were employed to analyze the captured vibrational signals using MATLAB R2017a (MathWorks):

$$
\operatorname{STFT}\{x[n]\}(m, \omega) \equiv X(\tau, \omega)=\sum_{n=-\infty}^{\infty} x[n] w[n-m] e^{-j \omega n}
$$


where $x_{n}$ is the sequence of discretized time-domain signals to be transformed, $m$ is the time index, $\omega$ is the frequency, and $w_{n}$ is the sequence of discretized window functions.

The purpose of VMD is to decompose the input signal into a number of discrete subsignals, ${ }^{(21)}$ providing outcomes with higher resolution:

$$
\begin{aligned}
\min _{\left\{u_{k}\right\},\left\{\omega_{k}\right\}}\left\{\sum_{k}\left\|\partial_{t}\left[\left(\delta(t)+\frac{j}{\pi t}\right) * u_{k}(t)\right] e^{-j \omega_{k} t}\right\|_{2}^{2}\right\}, \\
\text { s.t. } \quad \sum_{k} u_{k}=f,
\end{aligned}
$$

where $\left\{u_{k}\right\}:=\left\{u_{1}, \ldots, u_{K}\right\}$ and $\left\{\omega_{k}\right\}:=\left\{\omega_{1}, \ldots, \omega_{K}\right\}$ are shorthand notations of all modes and their center frequencies, respectively. $\sum_{k}:=\sum_{k=1}^{K}$ is the summation over all nodes.

To holistically examine the relationship between the vibrational characteristics of the sigmoid plate/vessel wall and PT, the confocal laser was focused at 1) the juxta-dehiscent sigmoid plate and the center of the exposed vessel wall before the separation procedure (step 1);2) the vessel wall after the separation procedure (step 2); and 3) the fixated hardened gelatin sponge smeared with surgical glue after the elimination of PT (step 3). The three steps are shown in Fig. 2. While

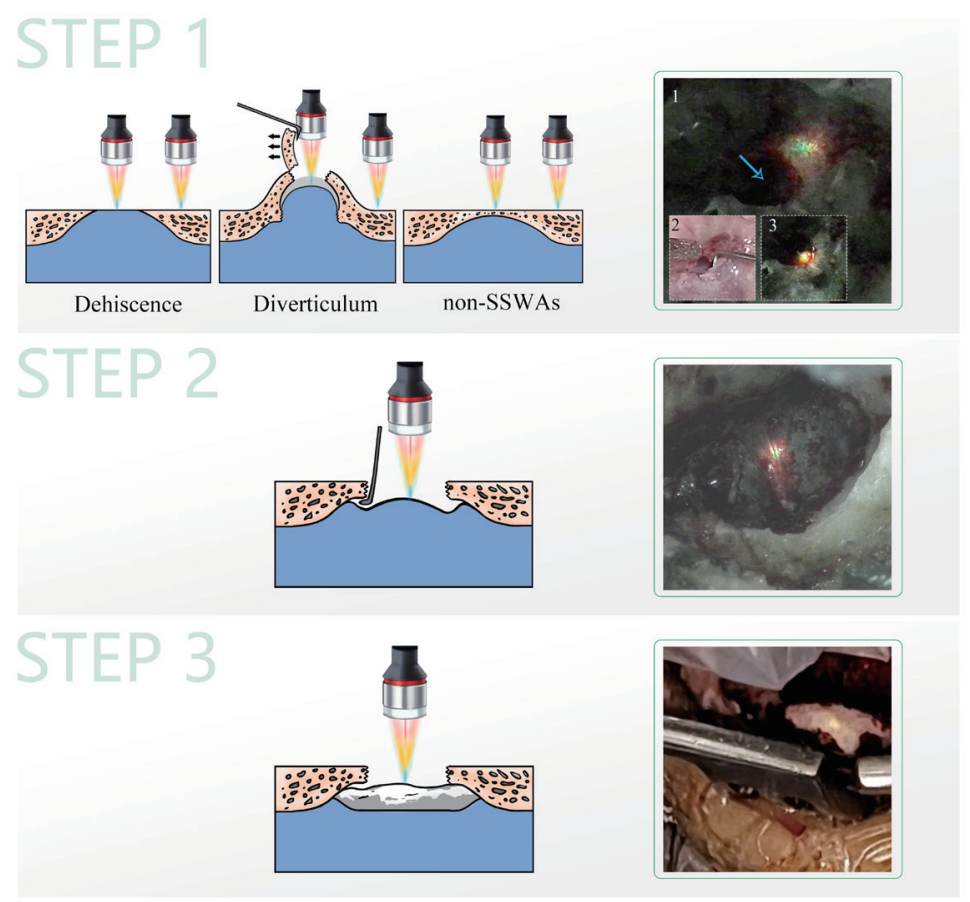

Fig. 2. (Color online) Intraoperative deployment of the confocal laser displacement sensor and the measurement design. (Step 1) Measurement of the sigmoid plate and focal area of the three groups (blue arrow indicates diverticulum). The intraoperative photographs showcase the (1) measurement of the sigmoid plate, (2) careful exposure of the dome of the diverticulum, and (3) measurement of the displacement at the dome of the diverticulum in case 5. (Step 2) Measurement of the sinus vessel wall after separation procedure (separating the vascular surface from the osseous wall). (Step 3) Measurement of the compressed vessel wall fixated with the hardened gelatin sponge after elimination of PT. 
performing under local anesthesia, all participants were asked to respond to surgeons whether their PT altered during each surgical step, and the participants were requested to breathe as little as possible during measurements.

\subsection{Doppler ultrasonographic detection and spectro-temporal analysis of venous flow sound}

All participants were asked to lie in the supine position with a neutral head position in a soundproof examination room for the ultrasonographic examination. The outflow characteristics of the bilateral dural venous sinus were detected using a color-coded Doppler ultrasonographic system (Esoate My Lab Class C, Italy). An LA-523 ultrasonographic transducer with a center frequency of 4-13 MHz was placed at the upper segment of the internal jugular vein at an angle near that of the mandible extending to the jugular bulb at skull base level (Fig. 1). The standardized 45 to $60^{\circ}$ insonation angle range was used. The sampling volume was adjusted to half of the luminal diameter while gradually proceeding from the near wall region to the center of the vascular lumen. Participants were instructed to inform the sonographer once the displaying sound best matched their PT. The subjective likeness of PT was rated using a visual analog scale (VAS). The matched flow sound was then picked up by an $\mathrm{H} 2 \mathrm{~N}$ handy recorder system (ZOOM, Japan) at 24-bit/48 kHz. The acoustic data were analyzed using MATLAB R2017a, similarly to in a previously described methodology. ${ }^{(22)}$ Low-pass filtering to $15 \mathrm{~Hz}$ using a tenth-order Butterworth filter was performed. The spectro-temporal analysis was carried out using a short-time Fourier transform. A Hanning window ( $\sim 50 \mathrm{~ms}, n=2048$ samples) was used with a hop size of $\sim 10 \mathrm{~ms}$ ( $n=512$ samples).

\subsection{Quantitative water occlusion test and intraoperative installment of piezoelectric film sensors}

A quantitative water occlusion test was performed to precisely evaluate the weight of the sterilized normal saline solution at body temperature used to eliminate PT. All participants were asked to lie with the ipsilesional ear towards the ceiling with a neutral head position. Warm saline solution was gently and slowly injected along the superior portion of the external auditory canal using a $5 \mathrm{~mL}$ syringe. The participants were asked whether their PT varied during the process. The amount of saline solution was documented at the precise moment when the loudness of PT changed. Changes in tone were not documented.

A ZD10-100 waterproof piezoelectric flexible thin-film sensor (K-Cut, China) was precalibrated and inserted between the osseous and vascular surfaces after the separation procedure in case 12. The response time of the sensor was set at $1 \mathrm{~ms}$ and the measuring range was $0-500$

g. The film sensor was disinfected by low-temperature plasma sterilization. The pressure data were collected using SSCOM software (K-Cut, China). 


\subsection{Computational flow field analysis}

We examined the correlation between the displacement of the sigmoid plate and the wall pressure exerted by flow impact. Patient-specific finite element models were established using Mimics 19.0 and 3-Matics 11.0 (Materialise, Belgium) for CFD simulations. Over 450000 tetrahedral elements and five layers of prismatic elements were established at the near wall regions adjacent to the vascular wall. All sinus branches with cross-sectional area under $1 \mathrm{~mm}^{2}$ were removed. The transient transverse-sigmoid flow field characteristics were investigated by solving the 3D transient Navier-Stokes equations and the continuity equation:

$$
\begin{gathered}
\nabla \cdot \boldsymbol{u}=0 \\
\rho \frac{\partial \boldsymbol{u}}{\partial t}+\rho \boldsymbol{u} \cdot \nabla \boldsymbol{u}=-\nabla \boldsymbol{p}+\nabla \cdot \tau+\rho \boldsymbol{g} .
\end{gathered}
$$

The transient $k-\omega$ turbulent model was selected to compute the flow field using Fluent 13.0 software (ANSYS, Inc., Cecil Township, PA):

$$
\begin{gathered}
\frac{\partial(\rho k)}{\partial t}+\frac{\partial\left(\rho u_{j} k\right)}{\partial x_{j}}=\rho P-\beta^{*} \rho \omega k+\frac{\partial}{\partial x_{j}}\left[\left(\mu+\sigma_{k} \frac{\rho k}{\omega}\right) \frac{\partial k}{\partial x_{j}}\right], \quad \text { with } P=\tau_{i j} \frac{\partial u_{i}}{\partial x_{j}} \\
\left.\frac{\partial(\rho \omega)}{\partial t}+\frac{\partial\left(\rho u_{j} \omega\right)}{\partial x_{j}}=\frac{\alpha \omega}{k} P-\beta \rho \omega^{2}+\frac{\partial}{\partial x_{j}}\left[\left(\mu+\sigma_{\omega} \frac{\rho k}{\omega}\right) \frac{\partial \omega}{\partial x_{j}}\right)\right]+\frac{\rho \sigma_{d}}{\omega} \frac{\partial k}{\partial x_{j}} .
\end{gathered}
$$

The velocity inlet was set at the transverse sinus entry using the patient-specific transient velocity profile; the zero-pressure outlet was located at the end of the sigmoid sinus at the level of the jugular foramen. A no-slip boundary condition was applied at the rigid wall of the vascular lumen. An incompressible Newtonian flow fluid, i.e., blood, with density $\rho=1050 \mathrm{~kg} / \mathrm{m}^{3}$ and dynamic viscosity $\mu=0.00345 \mathrm{~Pa} \cdot \mathrm{s}$ was established. The computing results were statistically averaged for 20 flow-pulsatory cycles.

\subsection{Statistical analysis}

The statistical analysis was performed using R version 3.4.3 (RStudio Version 1.1.383). The displacement and hydroacoustic (continuous) data were assessed for normality using the Shapiro-Wilk test. The null hypothesis that data came from a normally distributed population was rejected when $p<0.05$. The Levene and Kruskal-Wallis $\mathrm{H}$ tests were deployed to examine the outcomes of interest. A post-hoc comparison of the measured displacement and acoustic data of the three operative steps was performed using Dunn's test. Continuous variables are presented as the median and interquartile range (25/75\%). A linear regression model with one regressor was established to investigate the correlation between the sinus hydroacoustic parameters and the motion of the vessel wall. 


\section{Results}

\subsection{Temporal displacement of the sigmoid plate}

Table 1 and Fig. 3 summarize the temporal displacement data of the 12 participants. The short-time Fourier transform and VMD of case 1 are respectively shown in Figs. 4 and 5. The median sigmoid plate displacement of the 12 subjects was $0.0031(0 / 0.0056) \mathrm{mm}$, wherein those of the dehiscence, diverticulum, and non-SSWA groups were $0.0031(0.0008 / 0.0049), 0.0028$ $(0 / 0.0051)$, and $0.0038(-0.0026 / 0.0082) \mathrm{mm}$, respectively. The short-time Fourier transform

Table 1

Displacement data of all surgical procedures of the 12 participants. Variables are expressed as the median and interquartile range.

\begin{tabular}{|c|c|c|c|c|c|}
\hline & \multirow{2}{*}{ Procedures } & \multirow{2}{*}{$\begin{array}{l}\text { Location of } \\
\text { focus }\end{array}$} & \multicolumn{2}{|c|}{ SSWAs } & \multirow{2}{*}{$\begin{array}{c}\text { Non-SSWAs } \\
(n=3)\end{array}$} \\
\hline & & & Dehiscence $(n=5)$ & Diverticulum $(n=4)$ & \\
\hline \multirow{5}{*}{$\begin{array}{l}\text { Displacement } \\
(\mathrm{mm})\end{array}$} & \multirow{2}{*}{ Step 1} & $\begin{array}{l}\text { Sigmoid } \\
\text { Plate }\end{array}$ & $\begin{array}{c}0.0031 \\
(0.0008 / 0.0049)\end{array}$ & $\begin{array}{c}\S 0.0028 \\
(0 / 0.0051)\end{array}$ & $\begin{array}{c}0.0038 \\
(-0.0026 / 0.0082)\end{array}$ \\
\hline & & $\begin{array}{l}\text { Vessel } \\
\text { Wall\# }\end{array}$ & $\begin{array}{c}* 0.0013 \\
(-0.0082 / 0.0108)\end{array}$ & $\begin{array}{c}* \S 0.0174 \\
(0.0053 / 0.3548)\end{array}$ & NA \\
\hline & \multirow{2}{*}{ Step 2} & Vessel & 0.0458 & 0.0658 & 0.5514 \\
\hline & & Wall\# & $(0.0084 / 0.4549)$ & $(0.0127 / 0.7141)$ & $(0.0071 / 0.5413)$ \\
\hline & Step 3 & Compressed & $\begin{array}{c}-0.0054 \\
(-0.0098 /-0.0018)\end{array}$ & $\begin{array}{c}0.0007 \\
(-0.0035 / 0.0066)\end{array}$ & $\begin{array}{c}0.0015 \\
(0 / 0.0043)\end{array}$ \\
\hline
\end{tabular}

1. SSWAs indicates sigmoid sinus wall anomalies.

2. $\S$ indicates the statistical significance $(p<0.05)$ between the displacement of sigmoid plate and vascular wall before separation procedure.

3. * indicates the statistical significance $(p<0.05)$ of the displacement of the vascular wall between the dehiscence and diverticulum groups.

4. \# indicates the statistical significance $(p<0.05)$ before and after the separation procedure of all cohorts.

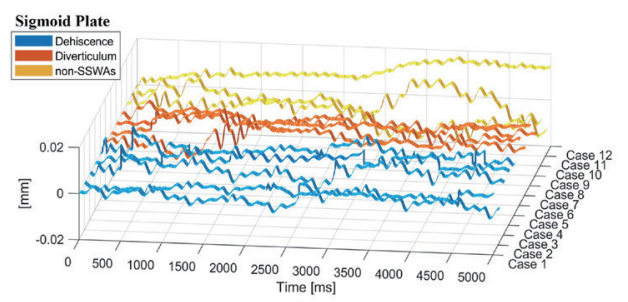

(a)

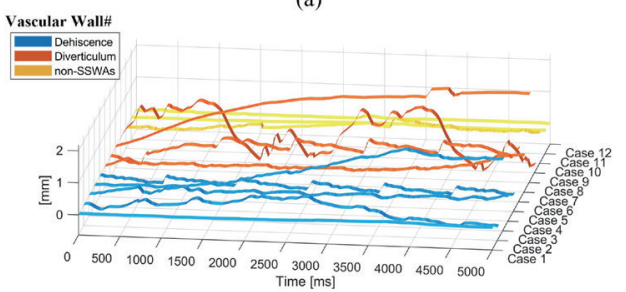

(c)

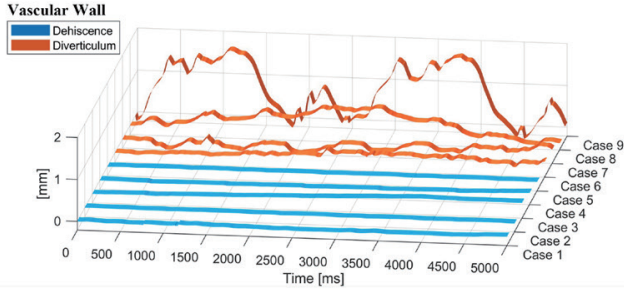

(b)

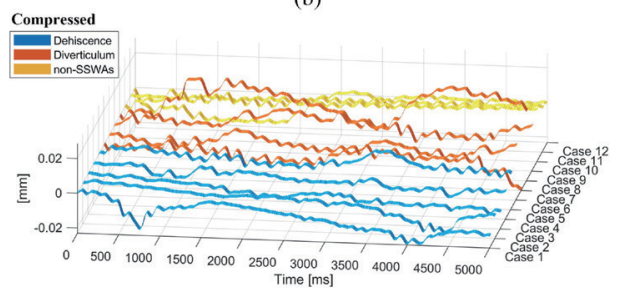

(d)

Fig. 3. (Color online) Vibration data of the 12 cases for $5 \mathrm{~s}$. (a) (step 1) Results of the sigmoid plate displacement. (b) (step 1) Results of the vessel wall displacement before the separation procedure. (c) (step 2) Results of the vessel wall displacement after the separation procedure. (d) (step 3) Results of the compressed and fixated vessel wall displacement after the elimination of PT. 


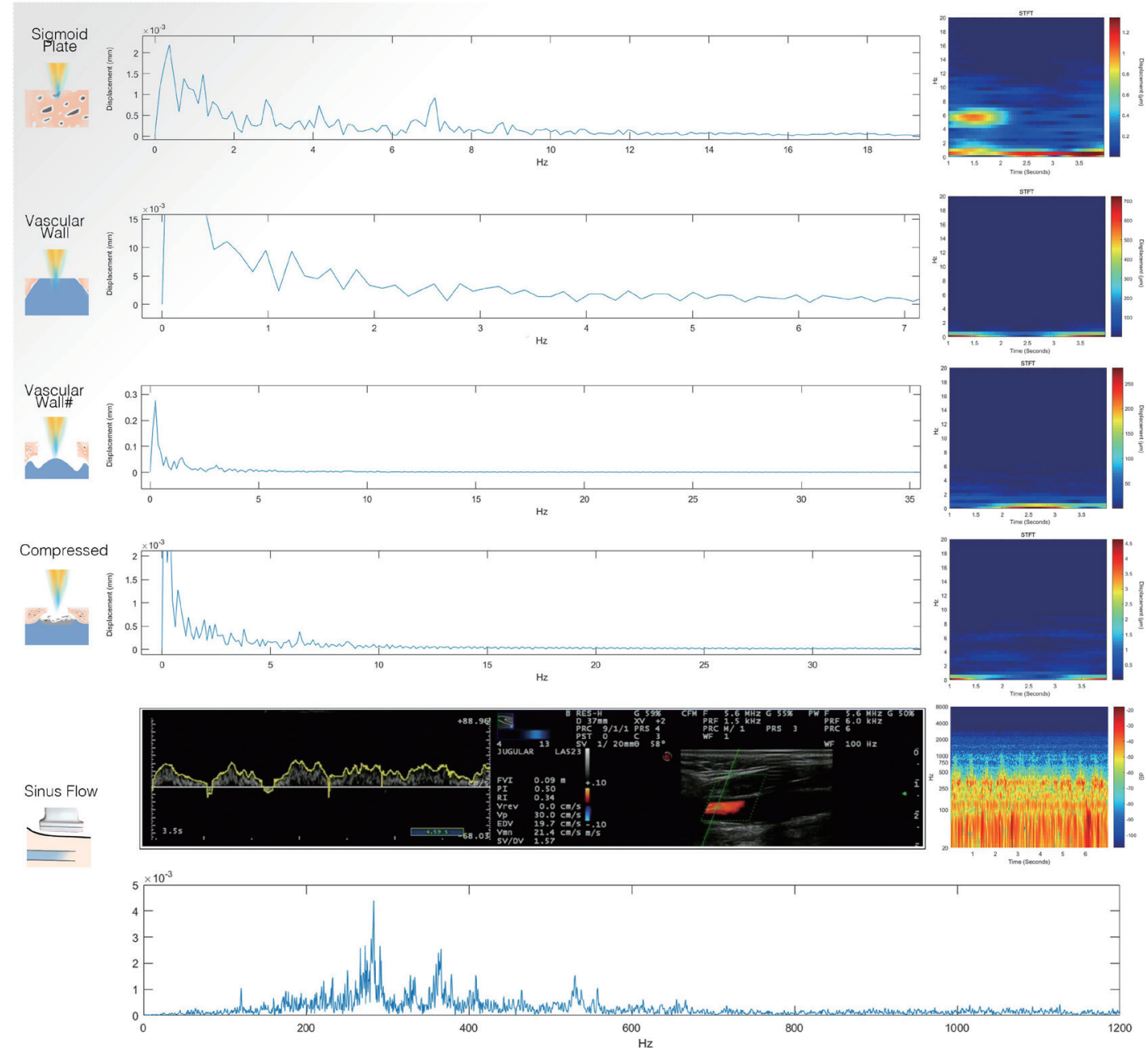

Fig. 4. (Color online) Results of short-time Fourier transform of the three steps in case 1. (step 1, sigmoid plate and vascular wall) Measurement of sigmoid plate and vessel wall before separation procedure, (step 2, vascular wall after separation procedure) measurement of the vessel wall after separation procedure, and (step 3, after compression technique) measurement of the compressed and fixated vessel wall after elimination of PT. The velocity spectrum and the profile of the ipsilesional upper neck venous flow at the center of the vascular lumen were detected by Doppler ultrasound. The spectro-temporal analysis showed that the frequency of PT at the peak amplitude was $278.4 \mathrm{~Hz}$ and that the frequency band of PT fluctuates at approximately $1000 \mathrm{~Hz}$.

showed that the median frequency at the peak displacement of the sigmoid plate was $1.37(1.15 / 5.42) \mathrm{Hz}$.

\subsection{Displacement of the vessel wall}

The vibration characteristics of the nine SSWA subjects were examined before the separation of the vessel wall from the osseous surface. The median displacements of the vessel wall of the dehiscence and diverticulum groups were $0.0013(-0.0082 / 0.0108)$ and $0.0174(0.0053 / 0.3548) \mathrm{mm}$, respectively. There was a statistical difference in the displacement of the vessel wall between the 


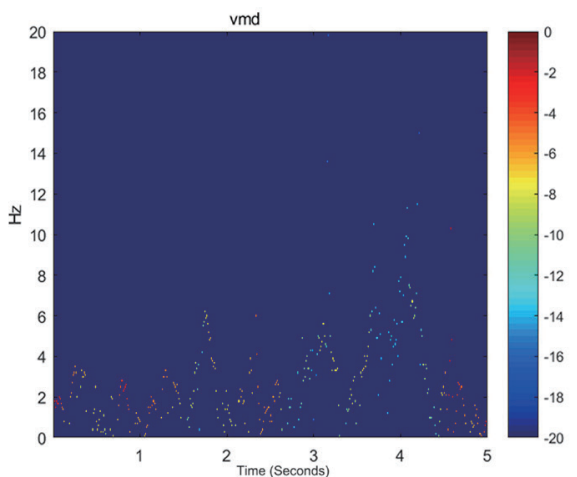

(a)

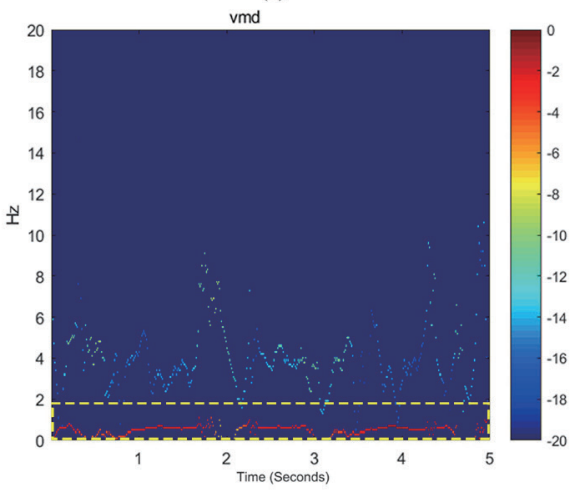

(c)

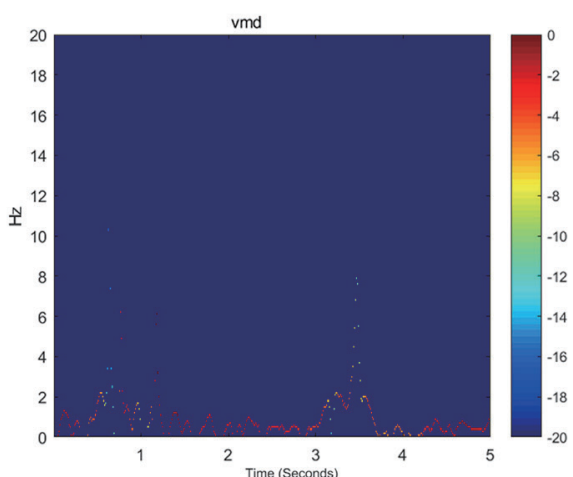

(b)

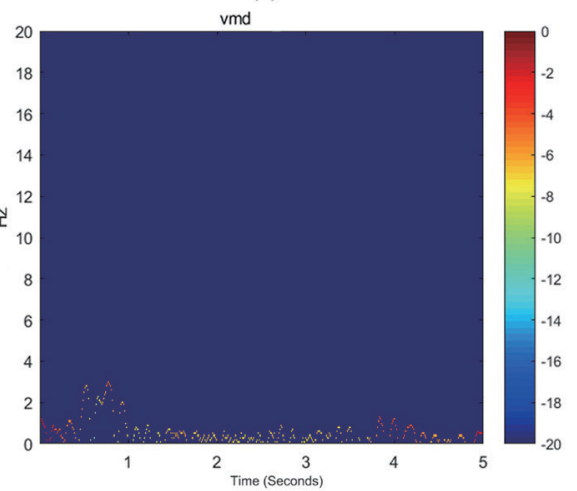

(d)

Fig. 5. (Color online) Results of VMD of the three surgical steps in case 1. Note that VMD clearly demonstrates the strong temporal displacement frequency of the vessel wall synchronous with heart diastole after separation from the osseous structure and dura mater (yellow rectangle) in comparison with the short-time Fourier transformation method. (a) (step 1) Displacement data of sigmoid plate. (b) (step 1) Displacement data of vascular surface before separation procedure. (c) (step 2) Displacement data after separation procedure. (d) (step 3) Displacement data after compression procedure.

diverticulum and dehiscence groups $(p<0.01)$, possibly due to the further protrusion of the diverticular wall that caused the overlying connective tissues, e.g., dura mater, to lose elasticity, although the displacement of the vascular wall in case 8 was significantly less than that for the intracohort diverticular cases. The median frequencies at the peak displacement of the vessel wall of the dehiscence and diverticulum were $2.2(1.4 / 2.8)$ and $1.8(0.8 / 7.9) \mathrm{Hz}$, respectively.

PT did not change in all participants after the vascular and osseous wall separation. The median of the vascular wall displacement after the separation procedure (step 2) was 0.0551 $(-0.0084 / 0.4841) \mathrm{mm}$. There was a statistical difference between the displacement before and after the separation procedure in both groups $(p<0.01)$, indicating that the displacement of the vascular wall only intensifies after the separation procedure. The median frequency at the peak displacement of the separated vessel wall of the 12 participants was $1.5(0.8 / 2.7) \mathrm{Hz}$.

PT was completely eliminated after the vessel wall was compressed and fixated in all 12 cases, and there were no postoperative implications during a two-month follow-up. The median displacement of the fixated structures was $-0.0012(-0.0067 / 0.0023)$. The median frequency at the peak displacement of the sigmoid plate was $1.51(0.91 / 4.84) \mathrm{Hz}$. 


\subsection{Hemodynamic outcomes and effects of vessel wall pressure}

The flow characteristics of the ipsilesional dural venous sinus outflow are shown in Table 2. The ipsilesional median peak and mean flow velocity were $0.373(0.303 / 0.652)$ and 0.315 $(0.237 / 0.461) \mathrm{m} / \mathrm{s}$, respectively. The sinus flow characteristics varied drastically among the subjects, where the mean pulsatility index was $0.375(0.212 / 1.177)$ and ranged from 0.16 to 2.10 . The spectro-temporal analysis demonstrated that the mean frequency range of the measured sinus flow sound was $0-1092.5(1023.5 / 1715.75) \mathrm{Hz}$. The median frequency at the peak amplitude of the 12 participants was $273.2(121.3 / 518.2) \mathrm{Hz}$. All participants reported that the displayed flow sound best matched their PT during the sampling of the flow at the center portion of the vascular lumen. The mean VAS score of the likeness of all patient-specific auscultated flow sounds was $9.5 \pm 0.645$.

The CFD results revealed that the mean peak sinus wall pressure probed at the tip of the transverse-sigmoid junction/diverticulum, mimicking the position of the laser focus spot, was $178.422(109.185 / 260.302)$ Pa. Linear regression analysis showed that the motion of the separated vessel wall peak had statistical significance with the wall pressure $(p=0.018)$ and flow velocity ( $p=0.0482$ ), as shown in Fig. 6. Among all the observed parameters, the wall pressure had the highest correlation with the displacement of the vessel wall $\left(r^{2}=0.443\right)$. The piezoelectric film sensor showed that the measured exerted force of case 12 was $2.065(1.92 / 2.18) \mathrm{g}$ with an insertion area of $0.25 \mathrm{~cm}^{2}$ (two sensor units). Figure 7 shows the outcomes calculated by CFD and measured by the piezoelectric film sensor.

\subsection{Quantitative water occlusion test}

In the quantitative water occlusion test, six of the nine SSWA participants reacted positively to the examination, whereas the technique was ineffective in all three non-SSWA participants. The median weight of the water injected into the external auditory canal was $1.4(1.1 / 2.4) \mathrm{g}$.

\section{Discussion}

This is the first study to numerically quantify the in vivo vibrational characteristics of sigmoid sinus plate dehiscence and the sinus vessel wall using a sophisticated confocal laser displacement sensor system. The current data indicate that PT is unlikely induced by the forced vibration of either the sigmoid plate or even the vessel wall, quantitatively bolstering recent

Table 2

Hemodynamic data of the 12 participants. Variables are expressed as the median and interquartile range.

\begin{tabular}{lcccccc}
\hline Variables & $\begin{array}{c}\text { Pulsatility } \\
\text { index }\end{array}$ & $\begin{array}{c}\text { Mean flow } \\
\text { velocity }(\mathrm{m} / \mathrm{s})\end{array}$ & $\begin{array}{c}\text { Peak flow } \\
\text { velocity }(\mathrm{m} / \mathrm{s})\end{array}$ & $\begin{array}{c}\text { Peak vessel wall } \\
\text { pressure }(\mathrm{Pa})^{\wedge}\end{array}$ & $\begin{array}{c}\text { Vascular } \\
\text { displacement\# }(\mathrm{mm})\end{array}$ & $\begin{array}{c}\text { Frequency range } \\
(\mathrm{Hz})\end{array}$ \\
\hline Variable & 0.375 & 0.3155 & 0.373 & 186.85 & 0.0551 & $0-1092.5$ \\
value & $(0.212 / 1.17)$ & $(0.2372 / 0.4617)$ & $(0.303 / 0.652)$ & $(99.227 / 267.330)$ & $(-0.0084 / 0.4841)$ & $(0-1023.5 / 0-1715.7)$ \\
\hline 1. \# indicates vascular displacement after the separation procedure (step 2). \\
2. ^ indicates the values obtained from CFD simulation.
\end{tabular}




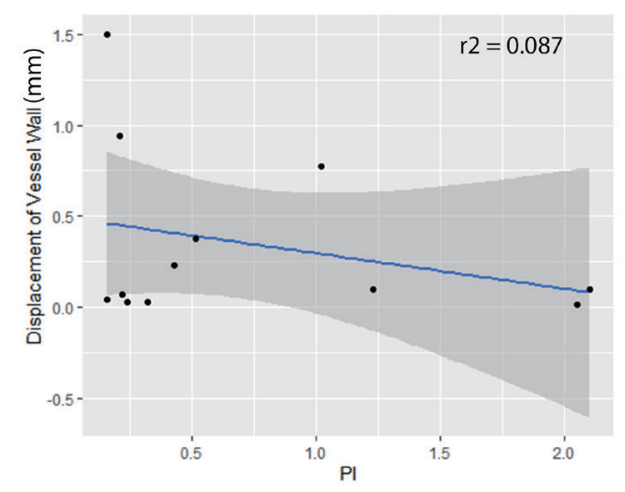

(a)

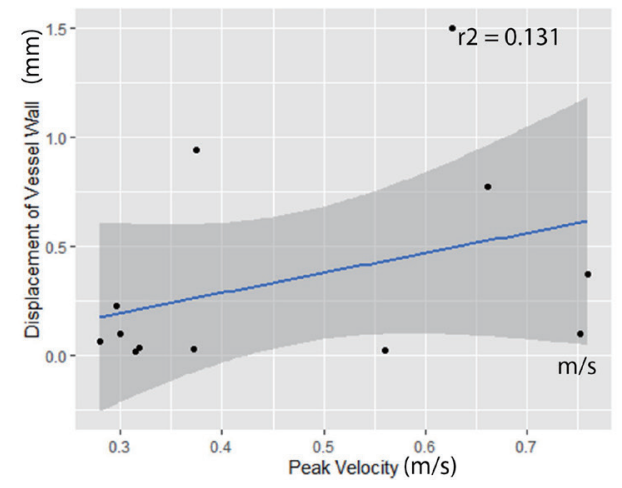

(c)

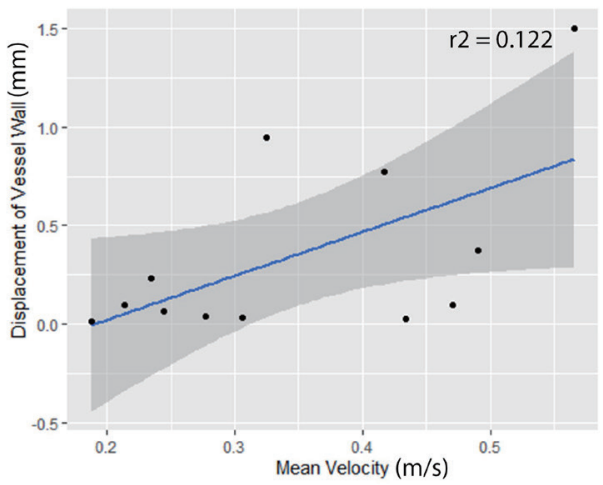

(b)

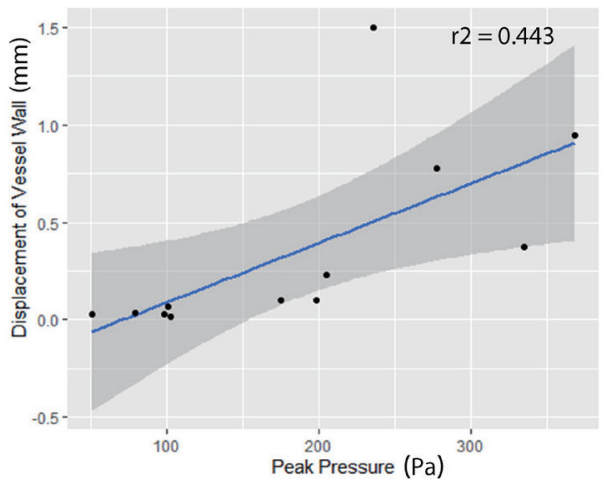

(d)

Fig. 6. (Color online) Results of univariate regression analysis showing the correlation between the displacement of the vessel wall and explanatory hemodynamic variables. (a) Correlation between vascular wall displacement and pulsatile index (PI). (b) Correlation between vascular wall displacement and mean velocity. (c) Correlation between vascular wall displacement and peak velocity. (d) Correlation between vascular wall displacement and peak pressure.

medical findings that the vibration of the exposed sigmoid sinus may not be a source of PT. ${ }^{(1,8,11)}$ There was no statistical difference between the displacement of the thinned sigmoid plate and the vessel walls in the dehiscence group (on average below $3.8 \mu \mathrm{m}$ ). This likely results from the resistance of the overlying ultrathin layer of the sigmoid plate or dura mater tightly fixated to the vessel wall. The current in vivo data suggest that $\mathrm{PT}$ associated with a dehiscent sigmoid plate arises mainly from Theory $A$ (mentioned in Sect. 1). If so, then a large proportion of patients with venous PT present an intact sigmoid plate, and in such cases, the fluid-borne sound caused by the pathological flow development may be a more dominant factor causing PT. This inference is highly plausible since the blood flow sound sampled by the ultrasonographic transducer closely matched the subjective perception of PT in all of our subjects, and the generated fluid sound most likely originates from the vortices at the transverse-sigmoid junction observed by intraoperative Doppler according to our previous report. ${ }^{(8)}$ In line with a recent case-series study using a trans-canal recording technique, the frequency at the peak PT amplitude in our subjects was near $250 \mathrm{~Hz},{ }^{(23)}$ and the harmony of the frequency range extended up to $1000-1500 \mathrm{~Hz}$. More importantly, the detected displacement frequency of the bone and vascular structures was 

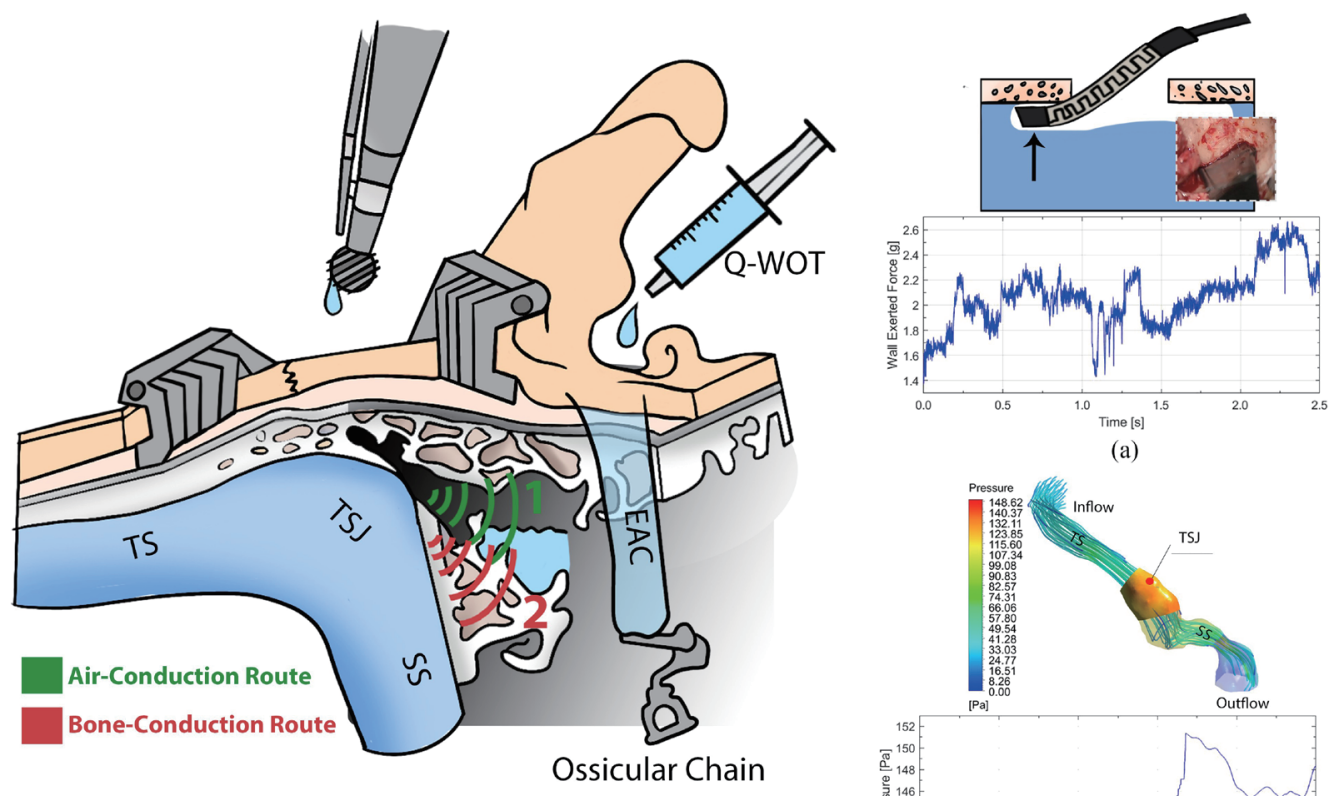

(a)

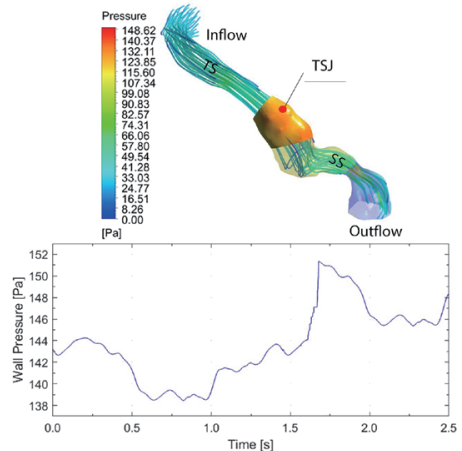

(b)

Fig. 7. (Color online) Green and red schematic sound waves (1) and (2) respectively indicate Theory A and Theory $B$ (mentioned in Sect. 1). PT persists after the entry of intraoperative fluid or the preclusion of the pulsating sinus wall. The sound wave energy or the flow-induced vibrational energy likely affects the tympanic membrane and the ossicular chain via both air/bone conduction routes. Q-WOT: quantitative water occlusion test; EAC: external auditory canal; TSJ: transverse-sigmoid junction; TS: transverse sinus; SS: sigmoid sinus. (a) Flow-exerted force measured by the calibrated piezoelectric film sensor $\left(0.25 \mathrm{~cm}^{2}\right)$ inserted between the osseous and vascular walls and (b) results of time-dependent wall pressure calculated by CFD.

primarily below $20 \mathrm{~Hz}$, which implies that the sigmoid plate most likely fails to preclude the sound transmission of the pathological aggregation of the sinus blood flow rather than being a source of sound production per se. Such a postulation can also illuminate that venous PT can be heard extrinsically, ${ }^{(6)}$ albeit not often, by an observer even through their bare ears without any perceivable vibrations manifested at the post-auricular mastoid region.

Although PT caused by the focal vibration of dehiscence alone seems unlikely, it would certainly be arbitrary to deny the possibility of the temporal bone as a medium for the transmission of PT. The longer wavelength of the low-frequency sounds creates resonance/vibration in walls, which helps propagate the passage of generated fluid sounds. Instead of the vibration-induced sound, it is likely that the vibrotactile sensation triggered by sinus flow may play a role, although the scale of the vibration may not be detectable using the current sensor system; it is also questionable if such a vibrotactile intensity is strong enough to be sensed by the organ of Corti. This notion is also supported by a recent audiometric study that found contralateral hearing threshold improvement after successful transmastoid surgery in a few cases. ${ }^{(10)}$ Although it was mentioned earlier that the blood flow sound may be a dominant factor inducing PT, the displacement of the entire sigmoid plate may also induce the sound 
production of PT. Such vibrational motion can be eliminated by reducing the wall pressure via an extraluminal compression technique or by thickening the sigmoid wall to prevent sound transmission and/or vibration via resurfacing surgery. ${ }^{(1,17)}$

The area of the exposed transverse-sigmoid junction vessel wall is generally miniscule at the focal dehiscent site, where its vibrational motion is intensified when a larger area of the above osseous structure and dura mater is detached. ${ }^{(7,8)}$ Even after the exposure of epidural space, the motion of the vascular/diverticular walls is unlikely to generate sound with frequency high enough to be heard. When performing transmastoid surgery under local anesthesia, the amplitude of PT does not variate cotemporally, precluding the motion of the (diverticular) vessel wall. ${ }^{(7,8,18)}$ Because PT persists even after opening up the mastoid cavity and the entry of fluids, it is also questionable whether the sound production due to vascular motion is amplified within the mastoid cavity, although PT can be eliminated by applying pressure to the tympanic membrane using the water occlusion test. ${ }^{(24)}$ In this study, the proposed quantitative water occlusion test eliminated PT in six of the nine subjects with SSWAs, whereas it was ineffective for the subjects without SSWAs. This technique is less consistent with regard to the clinical diagnostic value than jugular vein compression, which also possibly increases the PT amplitude. (24) Nevertheless, the current quantitative water occlusion test has the merit of revealing to surgeons the magnitude of the mechanical force produced by PT, which can be potentially indicative for transmastoid surgeries. However, both the destruction of the airtight mastoid cavity and the preclusion of the vascular wall motion failed to isolate PT in most of our reported patients, ${ }^{(7,8)}$ suggesting that PT may not be solely limited to the dynamic change of intra-mastoid air pressure and the motion of vessel walls. Together, it can be further inferred from these data that the sound wave exiting from a thinned/dehiscent sigmoid plate may not only transmit to the inner ear but also impact on the tympanic membrane and ossicular chain aerially. Furthermore, the transmission of the undetectable vibrational energy to the inner ear via bone conduction is highly suspected, which is evidenced by the fact that an intact sigmoid plate can be found in the same demographics. ${ }^{(8)}$

As this is the first study to deploy multiple sensor systems for the in vivo investigation of venous PT, some limitations and pitfalls may exist. Firstly, the small sample size due to the demanding intraoperative establishment (angulation/fixation) of sensors cannot fully expose outliers, although a general vibrational pattern of the PT-associated anatomical structures has been clearly displayed in the current study. According to the authors' clinical experiences, there are certainly cases with strong vascular wall pulsations, though seldomly encountered, that are not included in this study. For participants undergoing general anesthesia (excluded from this study), the measured displacement data are greatly affected by the intubation ventilator, for which the interference waves should be appropriately filtered. Secondly, despite the fact that the current confocal displacement sensor is the most sophisticated one to be established in both the clinical and experimental literature of PT to our knowledge, the accuracy of measurements is limited to the specifications of the sensors. Whether undetectable vibrations below $0.72 \mu \mathrm{m}$ that transmit via the air/bone conduction route play a role in causing PT remains unclear. Lastly, the data measured by the piezoelectric film sensor were pre-calibrated according to the reference weight, and thus, the gradient of the force exerted on the sigmoid plate should be of greater 
reference value than the actual value per se. Further analyses on the autocorrelation and periodicity of measured vibrational/acoustic data and their correlation with the results of the quantitative water occlusion test using larger study samples are warranted.

\section{Conclusions}

According to the data gauged by the current intraoperative confocal laser sensor system, the frequency of the regional displacement of the sigmoid plate and sinus vessel wall is predominantly below $20 \mathrm{~Hz}$. Whether vibrations below $0.72 \mu \mathrm{m}$ (beyond current detection) in the frequency range above $20 \mathrm{~Hz}$ contribute to the mechanical production of PT requires further examination. Instead of vibrational sound production, the Doppler ultrasonographic outcomes indicate that PT most likely results from flow-induced sounds sampled at the luminal center of the sinus lumen. If the cochlear sensitivity is indeed unaltered, it is therefore plausible that PT originates from a change in sinus hemodynamics, particularly the alteration of the total dural venous sinus flow volume and/or regional pressure gradient of the sinus flow. Of note, the persistence of PT after the destruction of the airtight condition with fluids entering into the mastoid cavity contradicts the disappearance of PT during the quantitative water occlusion test (with approximately 1-2 g of external force). Thus, the vibrotactile effect arising from flowinduced energy transmitted via air/bone conduction on the tympanic membrane and/or ossicular chain can be a critical yet overlooked concomitant mechanistic factor contributing to PT.

\section{Acknowledgments}

WW was supported by the National Natural Science Foundation of China (NSFC no. 81670933). There are no financial disclosures or conflicts of interest for either of the authors. The authors thank Xiaobing Xu, Ph.D., for Doppler ultrasound examinations. All illustrations were designed and produced by Y-LH. The recorded PT acoustic data can be shared upon reasonable request to Y-LH and WW.

\section{References}

1. D. J. Eisenman, P. Raghavan, R. Hertzano, and R. Morales: Laryngoscope 128 (2018) S1. https://doi. org/10.1002/lary.27218

2 D. J. Eisenman: Otol. Neurotol. 32 (2011) 1116. https://doi.org/10.1097/MAO.0b013e31822a1c7d

3 P. Zhao, H. Lv, C. Dong, Y. Niu, J. Xian, and Z. Wang: Eur. Radiol. 26 (2016) 9. https://doi.org/10.1007/s00330015-3827-8

4 A. Sismanis: Otolaryngol. Clin. North. Am. 36 (2003) 389. https://doi.org/10.1097/MAO.0b013e31822alc7d

5 A. Sismanis: Curr. Opin. Otolaryngol. Head Neck Surg. 19 (2011) 348. https://doi.org/10.1097/ MOO.0b013e3283493fd8

6 A. Sismanis: Laryngoscope. 97 (1987) 1. https://doi.org/10.1288/00005537-198708001-00001

7 Y. L. Hsieh and W. Q. Wang: Otol. Neurotol. 41 (2020) e132. https://doi.org/10.1097/MAO.0000000000002464

8 Y. L. Hsieh, X. B. Xu, Y. D. Hsieh, Y. C. Hsieh, D. Wang, P. Ping, and W. Q. Wang: Auris Nasus Larynx (2021). https://doi.org/10.1016/j.anl.2021.01.013

9 A. C. Wang, A. N. Nelson, C. Pino, P. F. Svider, R. S. Hong, and E. Chan: Otol. Neurotol. 38 (2017) 1390. https://doi.org/10.1097/MAO.0000000000001612

10 P. Raghavan, Y. Serulle, D. Gandhi, R. Morales, K. Quinn, K. Angster, R. Hertzano, and D. Eisenman: Am. J. Neuroradiol. 37 (2016) 136. https://doi.org/10.3174/ajnr.A4511 
11 K. Bhatnagar, A. T. Lataille, and D. J. Eisenman: Am. J. Otolaryngol. 41 (2020) 102647. https://doi. org/10.1016/j.amjoto.2020.102647

12 R. Zeng, G. P. Wang, Z. H. Liu, X. H. Liang, P. F. Zhao, Z. C. Wang, and S. S. Gong: PLoS One 11 (2016) e0164728. https://doi.org/10.1371/journal.pone.0164728

13 C. S. Kim, S. Y. Kim, H. Choi, J. W. Koo, S. Y. Yoo, G. S. An, K. Lee, I. Choi, and J. J. Song: J. Neurosurg. 125 (2016) 441. https://doi.org/10.3171/2015.6.JNS15961

14 G. P. Wang, R. Zeng, Z. H. Liu, X. H. Liang, J. F. Xian, Z. C. Wang, and S. S. Gong: Acta Otolaryngol. 134 (2014) 7. https://doi.org/10.3109/00016489.2013.831479

15 K. J. Otto, P. A. Hudgins, W. Abdelkafy, and D. E. Mattox: Otol. Neurotol. 28 (2007) 48. https://doi. org/10.1097/01.mao.0000247814.85829.f6

16 J. J. Song, Y. J. Kim, S. Y. Kim, Y. S. An, K. Kim, S. Y. Lee, and J. W. Koo: Neurosurgery 77 (2015) 709. https://doi.org/10.1227/NEU.0000000000000902

17 G. S. Liu, B. C. Boursiquot, N. H. Blevins, and Y. Vaisbuch: Otolaryngol. Head Neck Surg. 160 (2019) 749. https://doi.org/10.1177/0194599818823205

18 P. Guo and W. Q. Wang: Clin. Exp. Otorhinolaryngol. 8 (2015) 111. https://doi.org/10.3342/ceo.2015.8.2.111

19 S. Tian, X. Fan, Y. Wang, Z. Liu, and L. Wang: J. Biomech. 84 (2019) 197. https://doi.org/10.1016/j. jbiomech.2018.12.049

20 S. C. Joseph, E. Rizk, and R. S. Tubbs: Anatomy, Imaging and Surgery of the Intracranial Dural Venous Sinuses, eds. R. S. Tubbs (Elsevier, New York, 2020) pp. 205-220. https://doi.org/10.1016/B978-0-323-65377$\underline{0.00025-8}$

21 K. Dragomiretskiy and D. Zosso: IEEE Trans. Signal Process. 62 (2014) 531. https://doi.org/10.1109/ TSP.2013.2288675

22 Y. L. Hsieh, X. B. Xu, P. Guo, and W. Q. Wang: Auris Nasus Larynx (2020). https://doi.org/10.1016/j. anl.2020.06.012

23 S. Y. Lee, M. K. Kim, Y. J. Bae, G. S. An, K. Lee, B. Y. Choi, J. W. Koo, and J. J. Song: Sci. Rep. 10 (2020) 18194. https://doi.org/10.1038/s41598-020-75348-3

24 S. N. Park, J. S. Han, J. M. Park, H. J. Jin, H. A. Joo, J. T. Park, J. Y. Koo, Y. Kim, M. Y. Kwak, J. W. Seo, C. I. Song, and H. J. Park: Clin. Otolaryngol. 45 (2020) 280. https://doi.org/10.1111/coa.13493

\section{About the Authors}

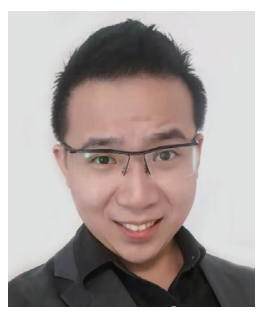

Yue-Lin Hsieh received his M.D. degree in 2019 and completed a three-year residency training program at the Fudan University-affiliated Eye \& ENT Hospital, Department of Otorhinolaryngology-Head and Neck Surgery from 2016 to 2019. He is currently a Ph.D. candidate of Fudan University. He is particularly dedicated to the clinical management and biomedical investigation of pulsatile tinnitus. Cerebrospinal fluid/dural venous sinus hemodynamics, endolymphatic hydrops, and middle-ear mechanics are also his research interests. (tb22521208@hotmail.com)

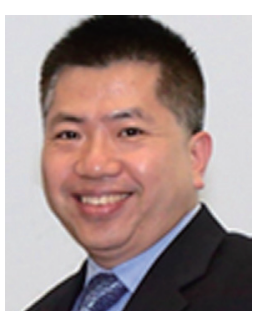

Wuqing Wang received his Ph.D. degree from the Fudan University-affiliated Eye \& ENT Hospital, Department of Otorhinolaryngology-Head and Neck Surgery, China, in 2003. He has been an associate professor of otolaryngologyhead and neck surgery and chief of endoscopic ear surgery, Division of Neurotology, and Skull Base Surgery of the Fudan University-affiliated Eye \& ENT Hospital since 2012. He is currently a leading otologic surgeon specializing in endoscopic ear surgery in China. He is a member of the Association for Research in Otolaryngology, American Academy of 
Otolaryngology-Head and Neck Surgery, The International Association of Physicians in Audiology, and the Bárány Society. His current research interests involve surgical management and biomedical investigation of chronic otitis media, Ménière's disease, vestibular migraine, endolymphatic hydrops, idiopathic sudden sensorineural hearing loss, and pulsatile tinnitus. (wwuqing@189.cn/wwuqing@eent.shmu.edu.cn) 